\title{
On Some Weak Monomorphisms and Weak Epimorphisms of Pro-HTop*
}

\author{
I. POP
}

\begin{abstract}
Related to Shape Theory, in a previous paper [6] we studied weak monomorphisms and weak epimorphisms in the category of pro-groups. In this note we give some intrinsic characterizations of the weak monomorphisms and the weak epimorphisms in pro-HTop" in the case when one of the two objects of such a morphism is a rudimentary system.
\end{abstract}

\section{INTRODUCTION}

If $\mathcal{C}$ is a category with zero-objects then a morphism $f: A \rightarrow B$ of $\mathcal{C}$ is a weak monomorphism if $f \circ u=0$ implies $u=0$. A morphism $f: A \rightarrow B$ is called a weak epimorphism if $u \circ f=0$ implies $u=0$.

Weakened versions of categorical notions of monomorphism and epimorphism have proved to be of some interest in pointed homotopy theory. A study of the comparison between weak monomorphism and monomorphism in homotopy theory was carried by T.Ganea [3] who, in

1991 Mathematics Subject Classification: 55 P5

Servicio publicaciones Univ. Complutense. Madrid, 1996. 
particular, obtained examples of weak monomorphisms which are not monomorphisms. Examples of homotopy weak monomorphisms which are not homotopy epimorphisms have been given by J.Roitberg [7]. Certainly, the study of shape monomorphisms and epimorphisms and their weakened versions can be interesting (see, for the homotopy case, the recently papers of E.Dyer \& J.Roitberg [2] and J.Dydak [1]). In [6] we characterized weak monomorphisms and weak epimorphisms in the category of pro-groups and we defined the notion of weakly exact sequence and we studied this notion in the category of pro-groups.

In this note we consider the pro-category of HTop*, the homotopy category of pointed topologal spaces, and we give some intrinsic characterizations of weak monomorphisms and weak epimorphisms $\underline{f}: \underline{X} \rightarrow \underline{Y}$ in pro-HTop ${ }^{*}$, when $\underline{X}$ or $\underline{Y}$ is a rudimentary system. These results can be interesting (and maybe sufficient) so a shape morphism $F: X \rightarrow Y$ between topological spaces $X$ and $Y$ can be given by means of such morphisms $f: \underline{X} \rightarrow \underline{Y}$ in pro-HTop* (approaching morphisms). The study of an arbitrary morphism $f: \underline{X} \rightarrow \underline{Y}$ of pro-HTop* is more complicated.

The notions and properties of pro-categories which are used in this paper are those of the book of S.Mardešić and J.Segal [4].

\section{WEAK MONOMORPHISMS IN THE CATEGORY PRO- HTOP*}

The category pro-HTop* is a category with zero objects. A zeroobject is a single point rudimentary system.

If $(X, *)$ is a rudimentary system in pro-HTop* and if $\underline{Y}=\left(\left(Y_{\lambda}, *\right)\right.$, $\left.q_{\lambda \lambda^{\prime}}, \Lambda\right)$ is an arbitrary object in pro-HTop ${ }^{*}$, then the morphisms $\underline{\underline{f}}=$ $\left(f_{\lambda}\right):(X, *) \rightarrow \underline{Y}$ coincide with the morphisms in inv-HTop*, the category of inverse systems in HTop* $[4$, p.20]. This means that for each $\lambda \in \Lambda$ is given a morphism $f_{\lambda}:(X, *) \rightarrow\left(Y_{\lambda}, *\right)$ in HTop* and for each pair $\lambda \leq \lambda^{\prime}$ we have $q_{\lambda \lambda^{\prime}} f_{\lambda^{\prime}}=f_{\lambda}$.

Lemma 1. For a morphism $\underline{E}:(X, *) \rightarrow \underline{Y}=\left(\left(Y_{\lambda}, *\right), q_{\lambda \lambda^{\prime}}, \Lambda\right)$ in pro-HTop* ${ }^{*}$ there exist an object $\underline{P}=\left(\left(P_{\lambda}, *\right), r_{\lambda \lambda^{\prime}}, \Lambda\right)$ and two morphisms $\underline{\underline{p}}=\left(p_{\lambda}, 1_{\Lambda}\right): \underline{P} \rightarrow \underline{Y}, \underline{h}=\left(h_{\lambda}\right):(X, *) \rightarrow \underline{P}$ such that for each $\lambda \in \Lambda$ :

(i) $h_{\lambda}:(X, *) \rightarrow\left(P_{\lambda}, *\right)$ is a pointed homotopy equivalence, 
(ii) $p_{\lambda}:(P, *) \rightarrow\left(Y_{\lambda}, *\right)$ is a pointed fiber map,

(iii) $f_{\lambda}=p_{\lambda} \circ h_{\lambda}$.

Proof. The existence for each $\lambda \in \Lambda$ of a factorization (iii), satisfying (i) and (ii), is well known $[5, p .249]$. For a pair $\lambda \leq \lambda^{\prime}$ in $\Lambda$ we define $r_{\lambda \lambda^{\prime}}=h_{\lambda} \circ h_{\lambda^{\prime}}^{-1}$, for which is immediate that $\underline{P}=\left(\left(P_{\lambda}, *\right), r_{\lambda \lambda^{\prime}}, \Lambda\right)$ is an inverse system in HTop ${ }^{*}$ and that $\underline{h}=\left(\overline{h_{\lambda}}\right):(X, *) \rightarrow \underline{\underline{P}}$ is a morphism in pro-HTop*. Also, from the relations $q_{\lambda \lambda^{\prime}} \circ f_{\lambda^{\prime}} \approx f_{\lambda}, f_{\lambda}=$ $p_{\lambda} h_{\lambda}, f_{\lambda^{\prime}}=p_{\lambda^{\prime}} \circ h_{\lambda^{\prime}}$, we deduce that $q_{\lambda \lambda^{\prime}} p_{\lambda} \approx p_{\lambda^{\prime}} \circ r_{\lambda \lambda^{\prime}}$, which shows that $\underline{\underline{p}}=\left(p_{\lambda}, 1_{\Lambda}\right): \underline{\underline{P}} \rightarrow \underline{\underline{Y}}$ a morphism of pro-HTop*.

Remark 1. It is obvious from Lemma 1 that we can write the equality $\underline{\underline{f}}=\underline{p} \circ \underline{h}$, in the category pro-HTop*, where $\underline{h}$ is an isomorphism. Then it is clear that $f$ is a weak monomorphism if and only if $p$ is a weak monomorphism. We will refer to the morphism $\underline{p}: \underline{\underline{P}} \rightarrow \underline{\underline{Y}}$ as the fibred factor of the morphism $\underline{f}$.

Remark 2. If $p=\left(p_{\lambda}, 1_{\Lambda}\right): \underline{P}=\left(\left(P_{\lambda}, *\right), r_{\lambda \lambda^{\prime}}, \Lambda\right) \rightarrow \underline{Y}=$ $\left(\left(Y_{\lambda}, *\right), p_{\lambda \lambda^{\prime}}, \Lambda\right)$ is a fibred factor in pro-HTop*, we can consider the fiber of this morphism by the object $\underline{F}=\left(\left(F_{\lambda}, *\right), r_{\lambda \lambda^{\prime}}^{\prime}, \Lambda\right)$, where $F_{\lambda}=p_{\lambda}^{-1}(*)$ and $r_{\lambda \lambda^{\prime}}^{\prime}=r_{\lambda \lambda^{\prime}} / F_{\lambda^{\prime}}$, for $\lambda \leq \lambda^{\prime}$. Then we can define a morphism $\underline{i}=\left(i_{\lambda}, 1_{\Lambda}\right): \underline{F} \rightarrow \underline{\underline{P}}$, where $i_{\lambda}$ is the inclusion of $\left(F_{\lambda}, *\right)$ in $\left(P_{\lambda}, *\right)$.

Definition 1. We will say that the fiber $\underline{F}=\left(\left(F_{\lambda}, *\right), r_{\lambda \lambda^{\prime}}^{\prime}, \Lambda\right)$ of the fibered factor $\underline{p}=\left(p_{\lambda}, 1_{\Lambda}\right): \underline{p}=\left(\left(P_{\lambda}, *\right), r_{\lambda \lambda^{\prime}}, \Lambda\right) \rightarrow \underline{Y}=$ $\left(\left(Y_{\lambda}, *\right), p_{\lambda \lambda^{\prime}}, \Lambda\right)$ is contractible in $\underline{P}$ if for each $\lambda \in \Lambda$ there is a $\lambda^{\prime} \geq \lambda$ such that $i_{\lambda} \circ r_{\lambda \lambda^{\prime}}^{\prime} \approx *$.

Theorem 1. A morphism $\underline{f}=\left(f_{\lambda}\right):(X, *) \rightarrow \underline{Y}$ is a weak monomorphism in the category pro-HTop* if and only if the fibre $E$ of every fibred factor $\underline{\underline{p}}=\underline{\underline{P}} \rightarrow \underline{\underline{Y}}$ is contractible in $\underline{\underline{P}}$.

Proof. By Remark 1 it is sufficient to prove that $\underline{p}$ is a weak monomorphism if and only if $\underline{\underline{F}}$ is contractible in $\underline{\underline{P}}$.

Suppose that $\underline{p}: \underline{\underline{P}} \rightarrow \underline{\underline{Y}}$ is a weak monomorphism in the category pro-HTop*. For the morphism $\underline{i}: \underline{\underline{F}} \rightarrow \underline{\underline{P}}$ from Remark 2 we have $p \circ \underline{i}=\underline{*}$ and by hypothesis it follows $\underline{i}=\underline{*}$. If $\underline{*}=(*, \Phi)$ then we have an equivalence $\left(i_{\lambda}, 1_{\Lambda}\right) \sim(*, \Phi)[4, \bar{p} .6]$ which implies that for each 
$\lambda \in \Lambda$ there is $\lambda^{\prime} \geq \lambda$ (and $\lambda^{\prime} \geq \Phi(\lambda)$ ) such that the following diagram in HTop* commutes

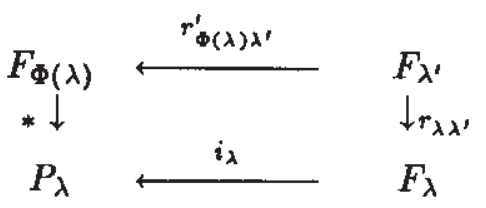

This implies that $i_{\lambda} \circ r_{\lambda \lambda^{\prime}}^{\prime}$ is pointed null-homotopic, i.e. $i_{\lambda} \circ r_{\lambda \lambda^{\prime}}^{\prime} \approx *$. Thus, $\underline{F}$ is contractible in $\underline{P}$.

Conversely, suppose that the libre $\underline{F}$ is contractible in $\underline{P}$ and let $\underline{\underline{u}}=$ $\left(u_{\lambda}, \Phi\right): \underline{Z}=\left(\left(Z_{\mu}, *\right), s_{\mu \mu^{\prime}}, M\right) \rightarrow \underline{P}=\left(\left(P_{\lambda}, *\right), r_{\lambda \lambda^{\prime}}, \Lambda\right)$ be a morphism, such that $\underline{p} \circ \underline{u}= \pm$. But $\underline{p} \circ \underline{u}=\left(p_{\lambda} \circ u_{\lambda}, \Phi\right)$, with the function $\Phi: \Lambda \rightarrow M$, and $p_{\lambda} \circ u_{\lambda}: Z_{\Phi(\lambda)} \rightarrow \bar{P}_{\lambda} \rightarrow Y_{\lambda}$. This relation implies that each $\lambda \in \Lambda$ admits $\mu \in M, \mu \geq \Phi(\lambda)$ such that $p_{\lambda} \circ u_{\lambda} \circ s_{\Phi(\lambda) \mu} \approx *$, by a pointed homotopy $H_{\lambda}: \bar{Z}_{\mu} \times[0,1] \rightarrow Y_{\lambda}$. Then, by the homotopy covering property of $p_{\lambda}$, there exists a pointed homotopy $K_{\lambda}: Z_{\mu} \times[0,1] \rightarrow P_{\lambda}$ such that $K_{\lambda}(\cdot, 0)=u_{\lambda} \circ s_{\phi(\lambda) \mu}$ and $p_{\lambda} \circ K_{\lambda}=H_{\lambda}$. Thus we have $u_{\lambda} \circ s_{\Phi(\lambda) \mu} \approx K_{\lambda}(\cdot, 1)$ and $\operatorname{Im} K_{\lambda} \subseteq F_{\lambda}$. By the proof of Lemma 1 and since the index sets are directed, for each $\lambda \in \Lambda$ we can choose the indices $\lambda^{\prime} \in \Lambda$ and $\mu, \mu^{\prime} \in M$ such that $i_{\lambda} \circ r_{\lambda \lambda^{\prime}} \approx *$ and the following diagram commutes

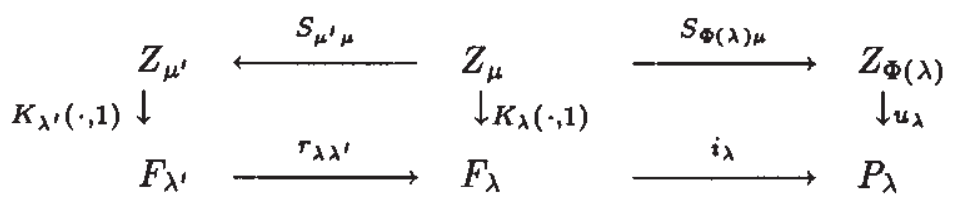

This means that $u_{\lambda} s_{\phi(\lambda) \mu} \approx i_{\lambda} r_{\lambda \lambda^{\prime}} K_{\lambda^{\prime}}(\cdot, 1) s_{\mu^{\prime} \mu} \approx *$, i.e. $\left(u_{\lambda}, \Phi\right) \sim$ $\left(*, \Phi^{\prime}\right)$ for satisfactory function $\Phi^{\prime}: \Lambda \rightarrow M$. Thus we obtained $\underline{u}=\underline{*}$, what finishes the proof of the theorem.

Remark 3. If $f:(X, *) \rightarrow(Y, *)$ is a pointed continuous map then $f$ is a weak monomorphism in HTop* if and only if it is a weak monomorphism in pro-HTop*. Theorem 1 generalizes the usual result for pointed continuous map [7, Prop. 2.2, (ii)]. 


\section{WEAK EPIMORPHISMS IN THE CATEGORY PRO- HTOP*}

In this section we consider only morphisms of the form $f: \underline{X} \rightarrow$ $(Y, *)$, where $\underline{X}$ is an arbitrary inverse system in HTop*. In fact the morphism $\underline{\underline{f}}$ can be represented by a continuous map $f_{\lambda}:\left(X_{\lambda}, *\right) \rightarrow$ $(Y, *)$, if $\underline{X}=\left(\left(X_{\lambda}, *\right), p_{\lambda_{\lambda^{\prime}}}, \Lambda\right)$, and two such maps $f_{\lambda_{1}}, f_{\lambda_{2}}$ define the same morphism $\underline{\underline{f}}$ if there is $\lambda \geq \lambda_{1}, \lambda_{2}$ such that $f_{\lambda} p_{\lambda_{1} \lambda}=f_{\lambda_{2}} p_{\lambda_{2} \lambda}$ in HTop*.

Lemma 2. For a morphism $\underline{\underline{f}}: \underline{X}=\left(\left(X_{\lambda}, *\right), p_{\lambda \lambda^{\prime}}, \Lambda\right) \rightarrow(Y, *)$ there exist an object $\underline{M}=\left(\left(M_{\lambda}, *\right), r_{\lambda \lambda^{\prime}}, \Lambda\right), \Phi: \Lambda^{\prime} \hookrightarrow \Lambda$ and two morphisms $\underline{j}=\left(j_{\lambda}, \Phi\right): \underline{X} \rightarrow \underline{M}, \underline{h}: \underline{M} \rightarrow(Y, *)$, such that for each $\lambda \in \Lambda^{\prime}$ :

(i) $h_{\lambda}:\left(M_{\lambda}, *\right) \rightarrow(Y, *)$ is a pointed homotopy equivalence,

(ii) $j_{\lambda}:\left(X_{\lambda}, *\right) \rightarrow\left(M_{\lambda}, *\right)$ is a pointed cofiber inclusion map,

(iii) $f_{\lambda}=h_{\lambda} \circ j_{\lambda}$.

Proof. Denote by $\Lambda^{\prime}$ the subset of $\Lambda$ such that an index $\lambda$ is in $\Lambda^{\prime}$ if and only if there is a map $f_{\lambda}:\left(X_{\lambda}, *\right) \rightarrow(Y, *)$ defining $\underline{f}$.

The existence for each $\lambda \in \Lambda^{\prime}$ of a factorization (iii) satisfying (i) and (ii) is well known [5, p.246]. For a pair $\lambda \leq \lambda^{\prime}$ in $\Lambda^{\prime}$ define $r_{\lambda \lambda^{\prime}}=$ $h_{\lambda}^{-1} \circ h_{\lambda^{\prime}}$, from which is immediate that $\left.\underline{M}=\left(M_{\lambda}, *\right), r_{\lambda \lambda^{\prime}}, \Lambda^{\prime}\right)$ is an inverse system in HTop ${ }^{*}$ and that all maps $h_{\lambda}, \lambda \in \Lambda^{\prime}$ define the same morphism $\underline{h}: \underline{M} \rightarrow(Y, *)$. Finally, if $\Phi: \Lambda^{\prime} \hookrightarrow \Lambda$ is the inclusion function, then $\underline{\underline{j}}=\left(j_{\lambda}, \Phi\right): \underline{X} \rightarrow \underline{M}$ is a morphism of pro-HTop*.

Remark 4. It is obvious from Lemma 2 that we can write $\underline{f}=\underline{h} \circ \underline{j}$, in the category pro-HTop ${ }^{*}$, where $\underline{h}$ is an isomorphism. Then it is clear that $\underline{f}$ is a weak epimorphism if and only if $\underline{j}$ is a weak epimorphism. We will refer to the morphism $\underline{j}: \underline{X} \rightarrow \underline{M}$ as the cofibred factor of the morphism $\underline{\underline{f}}$.

Remark 5. Let $\underline{j}=\left(j_{\lambda}, \Phi\right): \underline{X}=\left(\left(X_{\lambda}, *\right), p_{\lambda \lambda^{\prime}}, \Lambda\right) \rightarrow \underline{M}=$ $\left(\left(M_{\lambda}, *\right), r_{\lambda \lambda^{\prime}}, \Lambda^{\prime}\right)$ be a cofibred factor in pro-HTop*. Then for each $\lambda \in \Lambda^{\prime}$ we can consider the pointed quotient space $M_{\lambda} / X_{\lambda}$ with the pointed identification map $\pi_{\lambda}: M_{\lambda} \rightarrow M_{\lambda} / X_{\lambda}$. We can consider the 
inverse system $\underline{M} / \underline{X}=\left(\left(M_{\lambda} / X_{\lambda}, *\right), \bar{r}_{\lambda \lambda^{\prime}}, \Lambda^{\prime}\right)$ and the morphism $\underline{\pi}=$ $\left(\pi_{\lambda}, 1_{\Lambda^{\prime}}\right): \underline{M} \rightarrow \underline{M} / \underline{X}$. For the morphism $\underline{f}: \underline{X} \rightarrow(Y, *)$ we will say that $(Y, *)$ is contractible in the cofibred factor of $f$ if each $\lambda \in \Lambda^{\prime}$ admits a $\lambda^{\prime} \geq \lambda$ such that $\pi_{\lambda} \circ r_{\lambda \lambda^{\prime}} \approx *$.

Theorem 2. A morphism $f: \underline{X} \rightarrow(Y, *)$ of pro-HTop* is a weak epimorphism if and only if $(Y, *)$ is contractible in every cofibred factor.

Proof. Suppose that $f$ is a weak epimorphism, what is equivalent to the fact that the morphism $j: \underline{X} \rightarrow \underline{M}$ is a weak epimorphism. Since $\underline{\pi} \circ j= \pm$, the hypothesis implies $\pi= \pm$ in pro HTop*. This means $\left(\pi_{\lambda}, 1_{\Lambda^{\prime}}\right) \sim\left(*, \Phi^{\prime}\right)$, i.e. each $\lambda \in \Lambda^{\prime}$ admits a $\lambda^{\prime} \geq \lambda$ such that $\pi_{\lambda} \circ r_{\lambda \lambda^{\prime}}=*$ in HTop ${ }^{*}$. Thus, $(Y, *)$ is contractible in the cofibred factor $\underline{j}: \underline{X} \rightarrow \underline{M}$ of $\underline{f}$.

Conversely, suppose that $(Y, *)$ is coniractible in the cofibred factor of $\underline{f}$. It is sufficient to prove that $\underline{f}$ is a weak epimorphism. For this, suppose that for a morphism $\underline{u}=\left(\bar{u}_{\nu}, \Psi\right): \underline{M} \rightarrow \underline{Z}=\left(\left(Z_{\nu}, *\right), s_{\nu \nu^{\prime}}, N\right)$ we have $\underline{u} \circ j=\underline{*}$. This implies that for each $\nu \in N$ there is a pointed homotopy $H_{\nu}: u_{\nu} \circ j_{\Psi(\nu)} \approx *$. Then, by the pointed homotopy extension property of the pair $\left(M_{\Psi(\nu)}, X_{\Psi(\nu)}\right)$ there exists a pointed homotopy $K_{\nu}$ : $M_{\Psi(\nu)} \times[0,1] \rightarrow Z_{\nu}$, such that $K_{\nu}(\cdot, 0)=u_{\nu}$ and $K_{\nu} / K_{\Psi(\nu)} \times[0,1]=H_{\nu}$. Now, if we consider the pointed map $\varphi_{\nu}: M_{\Psi(\nu)} \rightarrow Z_{\nu}, \varphi_{\nu}=K_{\nu}(\cdot, 1)$, then we have $\varphi_{\nu} / X_{\Psi(\nu)}=K_{\nu} / X_{\Psi(\nu)} \times\{1\}=H_{\nu}(\cdot, 1)=*$. Therefore, we can define the pointed map $\tilde{\varphi}_{\nu}: M_{\Psi(\nu)} / X_{\Psi(\nu)} \rightarrow Z_{\nu}$, such that $\tilde{\varphi}_{\nu} \circ \pi_{\Psi(\nu)}=\varphi_{\nu}$ and the pointed homotopy $\tilde{\varphi}_{\nu} \circ F_{\Psi(\nu)}: M_{\lambda^{\prime}} \times[0,1] \rightarrow$ $M_{\Psi(\nu)} / X_{\Psi(\nu)} \rightarrow Z_{\nu}$, where $F_{\Psi(\nu)}: \pi_{\Psi(\nu)} \circ r_{\Psi(\nu) \lambda^{\prime}} \approx *$, for a convenient $\lambda^{\prime} \geq \Psi(\nu)$. For this we have $\tilde{\varphi}_{\Psi(\nu)} \circ F_{\Psi(\nu)}: \varphi_{\nu} \circ r_{\Psi(\nu) \lambda} \approx *$ in Top* ${ }^{*}$ On the other hand $K_{\nu}$ is a pointed homotopy, $K_{\nu}: u_{\nu} \circ r_{\Psi(\nu) \lambda} \approx \varphi_{\nu} \circ r_{\Psi(\nu) \lambda}$, and therefore $\tilde{\varphi}_{\nu} \circ F_{\Psi(\nu)} \circ K_{\nu}: u_{\nu} r_{\Psi(\nu) \lambda} \approx *$. This proves the equivalence $\left(u_{\nu}, \Psi\right) \sim(*, \Psi)$ for every $\nu \in N$ and therefore $\underline{u}= \pm$, what finishes the proof of the theorem.

Remark 6. If $f:(X, *) \rightarrow(Y, *)$ is a pointed continuous map then $f$ is a weak epimorphism in HTop* if and only if it is a weak epimorphism in pro-HTop*. Particularly, Theorem 2 generalizes the usual intrinsic characterization of a weak epimorphism in HTop* [7, Prop. 2.2 (i)]. 


\section{References}

[1] Dydak, J., Epimorphisms and monomorphisms in homotopy, Proc. of The Amer. Math. Soc., v. 116, 4 (1992), 1171-1173.

[2] Dyer, E. and Roitberg, J., Homotopy-epimorphisms, homotopy-monomorphisms and homotopy-equivalences, Topology and Its Appl. 46 (1992), 119-124.

[3] Ganea, T., On monomorphisms in homotopy theory, Topology, vol. 6 (1967), 149-152.

[4] Mardešić, S. and Segal, J., Shape Theory. The Inverse System Approach, North-Holland Publ. Com., 1982.

[5] Maunder, C.R.F., Algebraic Topology, Cambridge Univ. Press, 1980.

[6] Pop, I., On weak monomorphisms and weak epimorphisms in the category of pro-groups, Revista Matematica de la Univ. Complutense de Madrid, vol. 5, 2-3 (1992), 165-173.

[7] Roitberg, J., On weak epimorphisms in homotopy theory, Pacific J. of Math., vol. 121 (1986), 183-187. 